OPEN ACCESS

Edited by:

Abraham Wall-Medrano, Universidad Autónoma de Ciudad Juárez, Mexico

Reviewed by:

Claire L. Lyons,

Lund University, Sweden

Haifeng Zhao,

Shanxi Medical University, China

*Correspondence: Junbo Wang bmuwjbxy@bjmu.edu.cn

Specialty section:

This article was submitted to Nutrition and Metabolism, a section of the journal Frontiers in Nutrition

Received: 21 August 202 Accepted: 29 November 2021 Published: 24 December 2021

Citation:

Liu S, Yin X, Hou C, Liu X, Ma H, Zhang $X, X u M, X i e Y, L i Y$ and Wang $J$ (2021) As a Staple Food Substitute, Oat and Buckwheat Compound Has Health-Promoting Effects for Diabetic Rats. Front. Nutr. 8:762277. doi: 10.3389/fnut.2021.762277

\section{As a Staple Food Substitute, Oat and Buckwheat Compound Has Health-Promoting Effects for Diabetic Rats}

\author{
Siqi Liu ${ }^{1}$, Xueqian Yin ${ }^{1}$, Chao Hou ${ }^{1}$, Xinran Liu ${ }^{1}$, Huijuan Ma ${ }^{1}$, Xiaoxuan Zhang ${ }^{1}$, \\ Meihong $X u^{1}$, Ying $\mathrm{Xie}^{2}$, Yong $\mathrm{Li}^{1}$ and Junbo Wang ${ }^{1 *}$ \\ 1 Department of Nutrition and Food Hygiene, School of Public Health, Peking University, Beijing, China, ${ }^{2}$ Department of \\ Pharmaceutics, School of Pharmaceutical Sciences, Peking University, Beijing, China
}

Dietary intervention is crucial for the prevention and control of diabetes. China has the largest diabetic population in the world, yet no one dietary strategy matches the eating habits of the Chinese people. To explore an effective and acceptable dietary pattern, this study uses oat and buckwheat compound (OBC) as a staple food substitute and explored its effects on diabetic Sprague-Dawley rats. The model of diabetic rats was established by combining high-calorie feed and streptozotocin (STZ) injection. The dietary intervention for the seven groups, including a normal control group, a model control group, a metformin control group, a wheat flour control group, and three OBC groups with different doses, started from the beginning of the experiment and lasted for 11 weeks, two consecutive injections of STZ in small doses were operated the 6th week. General states, glucose metabolism, and lipid metabolism indexes were measured. Antioxidant and inflammatory indexes and pathologic changes of kidney and liver tissues were tested. Changes in kidney and ileum ultramicrostructure were detected. What's more, ileal epithelial tight junction proteins and gut microbiota were analyzed. Significant decreases in fasting blood glucose (FBG), glucose tolerance, serum insulin, and insulin resistance were observed in rats intervened with $\mathrm{OBC}$, and these rats also showed a higher level of superoxide dismutase (SOD) together with improved lipid metabolism, attenuated inflammation, and liver and kidney injuries. In addition, in OBC groups, the intestinal barrier was improved, and the disturbance of gut microbiota was reduced. These results suggest that $\mathrm{OBC}$ has health-promoting effects for diabetic rats, and since oat and buckwheat are traditionally consumed grains in China, OBC could be a potential and easy-to-accept staple food substitute for the dietary pattern for Chinese.

Keywords: oat, buckwheat, dietary intervention, staple food, diabetes, glucose metabolism, gut microbiota

\section{INTRODUCTION}

Diabetes is a serious chronic metabolic disease with complications in many parts of the body and can increase the overall risk of dying prematurely. Around 463 million people are estimated to be suffering from diabetes in 2019, which is expected to rise to 700 million (10.9\%) by 2,045 (1). The prevalence of diabetes in China is particularly serious. According to the International Diabetes 
Federation Diabetes Atlas 9th Edition, there are about 116 million diabetic patients in China, and 147 million are expected by 2,045 [International Diabetes Federation (IDF) Diabetes Atlas 2019].

According to the $\mathrm{WHO}$, a healthy diet plays a key role in reducing the risk of diabetes. Several dietary patterns such as the Mediterranean diet or patterns that replace all or part of the staple food with substances like potatoes or nuts have been demonstrated to reduce diabetes risk (2). However, the dietary preferences of Chinese people are quite different from Western countries. Grains account for a large part of the diet as the main staple food. As the increasing evidence revealed the predisposition to insulin resistance and diabetes due to traditional staple consumption of rice and wheat (3), oats and buckwheat, widely consumed grains in China, are better staple food substitutes. Oat and buckwheat had been proved to have significant effects on controlling hyperglycemia (4), insulin response (5), and diabetic kidney disease (6). In 2016, by taking a randomized control trial (RCT), Li (7) and others concluded that both short and long term oat intake (at least $50 \mathrm{~g}$ oats per day) had a significant effect on controlling hyperglycemia; similarly, another RCT showed that buckwheat intake by 110 $\mathrm{g} / \mathrm{d}$ could also improve insulin resistance in patients with type 2 diabetes mellitus (8). What's more, various research have shown that ingredients of oat as oat oligopeptides and oat $\beta$-glucan can exert a hypoglycemic effect $(9,10)$, as well as rutin and Dchiro-inositol in buckwheat could improve insulin sensitivity and regulate serum glucose level $(11,12)$. In addition, the correlation between diabetes and gut microbiota disorder has attracted increasing attention, and certain whole grains such as oat and barley, which are rich in dietary fibers, have also been shown to promote and maintain gut microbiota (13). However, due to the distinctive taste and texture, it is hard for people to consume one certain grain daily. By mixing two grains, the palatability could be improved. What's more, as single grains are usually not suitable as a staple food due to the lack of certain nutrients, especially amino acids, by mixing buckwheat and oat, amino acids could be complementary between buckwheat and oat, as buckwheat contains more lysine and arginine, and oat contains more glutamic acid, proline, and leucine.

In this work, we used oat and buckwheat compound (OBC) as a staple food substitute and explored its effects on diabetes by researching the physiology and gut microbiota of diabetic rats. This work will provide a valuable reference for promoting the exploration of dietary patterns that are suitable for the prevention and treatment of diabetes in China.

\section{MATERIALS AND METHODS}

\section{Materials and Reagents}

Oat and buckwheat compound is a mixture made from buckwheat and oat mixed in the ratio 2.2 to 1 . The ratio of buckwheat to oat was selected by our previous experiments. Both oat (Bayou 1) and buckwheat (Dasanleng) were purchased from DongFangLiang Life Technology Ltd. (Shanxi, China). The nutrients of oat and buckwheat used in the experiment are shown in Table 1, tested by the College of Food Science and Nutritional Engineering, China Agricultural University.
TABLE 1 | Nutrients of buckwheat and oat.

\begin{tabular}{lcc}
\hline Nutrients & Buckwheat & Oat \\
\hline Energy $(\mathrm{kJ} / 100 \mathrm{~g})$ & 1,407 & 1,574 \\
Protein $(\mathrm{g} / 100 \mathrm{~g})$ & 11.9 & 13.9 \\
Fat $(\mathrm{g} / 100 \mathrm{~g})$ & 2.8 & 8.0 \\
Carbohydrate $(\mathrm{g} / 100 \mathrm{~g})$ & 61.8 & 58.3 \\
Total dietary fiber $(\mathrm{g} / 100 \mathrm{~g})$ & 6.37 & 6.36 \\
Ash $(\mathrm{g} / 100 \mathrm{~g})$ & 1.6 & 1.5 \\
Water $(\mathrm{g} / 100 \mathrm{~g})$ & 15.5 & 11.9 \\
Vitamin $\mathrm{E}(\mathrm{mg} / 100 \mathrm{~g})$ & 7.08 & 1.22 \\
Phosphorus $(\mathrm{mg} / 100 \mathrm{~g})$ & 344 & 327 \\
Kalium $(\mathrm{g} / \mathrm{kg})$ & 4.36 & 3.54 \\
Manganese $(\mathrm{mg} / \mathrm{kg})$ & 12.6 & 40.4 \\
Selenium $(\mathrm{mg} / \mathrm{kg})$ & 0.0361 & 0.0311 \\
Quercetin $(\mathrm{g} / \mathrm{mg})$ & $<0.1$ & $<0.1$ \\
Ferulic acid $(\mu \mathrm{g} / 100 \mathrm{~g})$ & 1.01 & 29.9 \\
Protocatechuic acid $(\mu \mathrm{g} / 100 \mathrm{~g})$ & 14.6 & 10.6 \\
Vanillic acid $(\mu \mathrm{g} / 100 \mathrm{~g})$ & $<0.4$ & 3.59 \\
\hline & & \\
\hline
\end{tabular}

Streptozotocin (99.8\% purity) was purchased from Sigma Chemical Co. (St Louis, MO, USA). Glucose (99.8\% purity) was purchased from Beijing Jinhua Taiya Chemical Reagent Co., Ltd. (Beijing, China). Metformin hydrochloride extended-release tablets were purchased from Sino-American Shanghai Squibb Pharmaceuticals Ltd. (Shanghai, China, National medicine permit number: H20023370). Citric acid (99.9\% purity) and sodium citrate $(99.9 \%$ purity) used in the streptozotocin injection solution were purchased from Beijing Tong Guang fine chemicals company.

Basic feed was processed by Beijing Biotech HD Biotechnology Co., Ltd. (Beijing, China), according to the national standard (GB 14924.3-2010). High-calorie feed was processed by Beijing Biotech HD Biotechnology Co., Ltd. (Beijing, China) according to the evaluation method of auxiliary hypoglycemic function of health food (National Food and Drug Administration [2012]107). Feed for OBC groups and wheat flour control (WFC) group were made by replacing the cornstarch in high-calorie feed with different proportions of $\mathrm{OBC}$ or wheat flour (OBC content in each group feed: low dose: $29.3 \mathrm{~g} / \mathrm{kg}$, medium-dose: $58.7 \mathrm{~g} / \mathrm{kg}$, high dose: $117.3 \mathrm{~g} / \mathrm{kg}$ ). At the same time, maltodextrin, 10 in each group, had been adjusted to ensure that the feed quality of each group was equal, while calories of feeds for each group were in agreement (the differences were $<0.07 \%$ ). The feed ingredients of each group are shown in Table 2.

\section{Animal Housing Conditions}

Male Sprague-Dawley rats $(200 \pm 10 \mathrm{~g})$ were provided by the Department of Laboratory Animal Science, Peking University (Beijing, China) and bred in a specific pathogen free (SPF) room there, production certificate No.: SCXK (Beijing) 2016-0010, use license No.: SYXK (Beijing) 2016-0041. Two rats were housed per cage, with the temperature of the SPF room controlled at 
TABLE 2 | Feed ingredients of each group ( $g / \mathrm{kg})$.

\begin{tabular}{|c|c|c|c|c|c|c|c|}
\hline Group & Cornstarch & Wheat flour & OBC & Maltodextrin & Sucrose & Soybean oil & Lard \\
\hline NC & 298.6 & 0.0 & 0.0 & 33.2 & 331.7 & 23.7 & 19.0 \\
\hline $\mathrm{MC}$ & 84.8 & 0.0 & 0.0 & 116.5 & 201.4 & 29.1 & 206.8 \\
\hline WFC & 0.0 & 159.3 & 0.0 & 42.1 & 201.4 & 29.1 & 206.8 \\
\hline MPC & 84.8 & 0.0 & 0.0 & 116.5 & 201.4 & 29.1 & 206.8 \\
\hline OBC-L & 45.0 & 0.0 & 39.9 & 116.5 & 201.4 & 29.1 & 206.8 \\
\hline OBC-M & 5.1 & 0.0 & 79.7 & 116.5 & 201.4 & 29.1 & 206.8 \\
\hline OBC-H & 0.0 & 0.0 & 159.3 & 42.1 & 201.4 & 29.1 & 206.8 \\
\hline
\end{tabular}

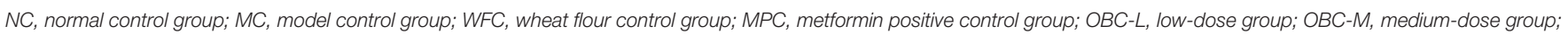
$\mathrm{OBC}-\mathrm{H}$, high-dose group.

$25^{\circ} \mathrm{C} \pm 1^{\circ} \mathrm{C}$, relative air humidity controlled at $50-60 \%$, and 12-h light/dark cycles. Due to the polyuria caused by diabetes, the cage bedding was changed daily after injecting STZ. All animal procedures meet the Regulation on the Administration of Laboratory Animals and were approved by the Ethics Committee of Peking University (ethics No.: LA2018189).

\section{Establishment of Diabetes Model}

Healthy adult male SD rats were fed adaptively for 1 week before the experiment. After 45 days fed by each group's corresponding feed, rats to be modeled received two intraperitoneal injections of low-dose STZ (30 mg/kg body weight) at an interval of 1 week. Three days after the second injection, rats were fasted for $5 \mathrm{~h}$ to measure their tail vein blood glucose. Rats with a blood glucose of $10-25 \mathrm{mmol} / \mathrm{L}$ were used as diabetic rats for our study.

\section{Experimental Design}

Based on their baseline serum glucose, 72 rats were randomly divided into seven groups, the normal control group (NC, $n=$ 10), the model control group (MC, $n=10$ ), the (WFC, $n=$ 10 ), the metformin positive control group (MPC, $n=10$ ), and three $\mathrm{OBC}$ treatment groups including a low dose group (OBCL, $n=10$ ), a medium dose group (OBC-M, $n=11$ ), and a high dose group (OBC-H, $n=11)$. As a reference hypoglycemic drug, metformin was used to intervene rats in the MPC group by gavage after the injection of STZ. The initial dose of metformin was $50 \mathrm{mg} / \mathrm{kg}$ body weight, and it increased by $50 \mathrm{mg} / \mathrm{kg}$ body weight every 2 weeks until the maximum dose at $200 \mathrm{mg} / \mathrm{kg}$ body weight. All rats in other groups received distilled water every day at the same time with a bodyweight volume of $10 \mathrm{ml} / \mathrm{kg}$ until the end of the experiment. The general state of rats includes haircoat state, mental state, body weight, water, and food intake were recorded daily. Six rats were randomly selected in each group and fed individually in the metabolic cage for $24 \mathrm{~h}$ to collect and record the urine volume of the rats. Each metabolic cage has a funnel and measuring cylinder for urine collection. The schematic diagram of the study protocol is shown in Figure 1.

\section{Determination of Fasting Blood Glucose and Oral Glucose Tolerance Test}

Fasting blood glucose levels of rats were measured every week. Rats fasted for $5 \mathrm{~h}$ before the FBG was measured through the tail vein. The oral glucose tolerance test was done on the $3 \mathrm{rd}, 7 \mathrm{th}$, and 11 th weeks of the experiment. After $12 \mathrm{~h}$ of fasting, the blood glucose of rats was measured by tail vein as the blood glucose level at $0 \mathrm{~h}$, and then rats were gavaged with $50 \%$ glucose solution at a dose of $2 \mathrm{~g} / \mathrm{kg}$ body weight, and blood glucose was recorded 0.5 , 1 , and $2 \mathrm{~h}$ after gavage. The area under the blood glucose curve of oral glucose tolerance test (OGTT-AUC) was calculated by the following formula:

$$
\begin{aligned}
O G T T-A U C= & 0.25 \times 0 \mathrm{hFBG}(\mathrm{mmol} / \mathrm{L})+0.5 \\
& \times 0.5 h F B G(\mathrm{mmol} / \mathrm{L})+0.75 \\
& \times 1 \mathrm{hFBG}(\mathrm{mmol} / \mathrm{L})+2 \mathrm{hFBG}(\mathrm{mmol} / \mathrm{L})
\end{aligned}
$$

Homeostasis model assessment of insulin resistance (HOMAIR) was calculated by the following formula:

$$
H O M A-I R=F B G(\mathrm{mmol} / L) \times \frac{I N S(\mu I U / m L)}{22.5}
$$

\section{Biochemical Analysis and Pathological Examination}

The main organs (kidney, liver, ileum, and caecum) of rats were removed and weighed at the end of the experiment to calculate the organ coefficients and for further testing including hematoxylin-eosin (HE) staining and ultramicrostructure observation. The level of serum insulin was detected by using an $\mathrm{XH}-6080 \gamma$ radioimmunocounter (Zhonghe, Xi'an, China). The level of SOD and malonaldehyde (MDA) were detected by using a 721-G Visible spectrophotometer (Jingke, Shanghai, China). The level of FBG and all other biochemical indicators include glycated serum protein, tumor necrosis factor- $\alpha$ (TNF- $\alpha$ ), interleukin-6 (IL-6), aspartate aminotransferase (AST), and alanine aminotransferase (ALT) were detected by using an AU480 automatic biochemistry analyzer (Olympus, Tokyo, Japan). The pathologic changes of kidney and liver tissues were detected by HE staining. Kidney ultramicrostructure was observed with a transmission electron microscope. Western blotting was used to detect ileal tight junction proteins. Cecal feces sample microbiota analysis was divided into the following sections: sample collection, DNA extraction and amplification, $16 \mathrm{~S}$ rDNA sequencing, and taxonomic classification. 


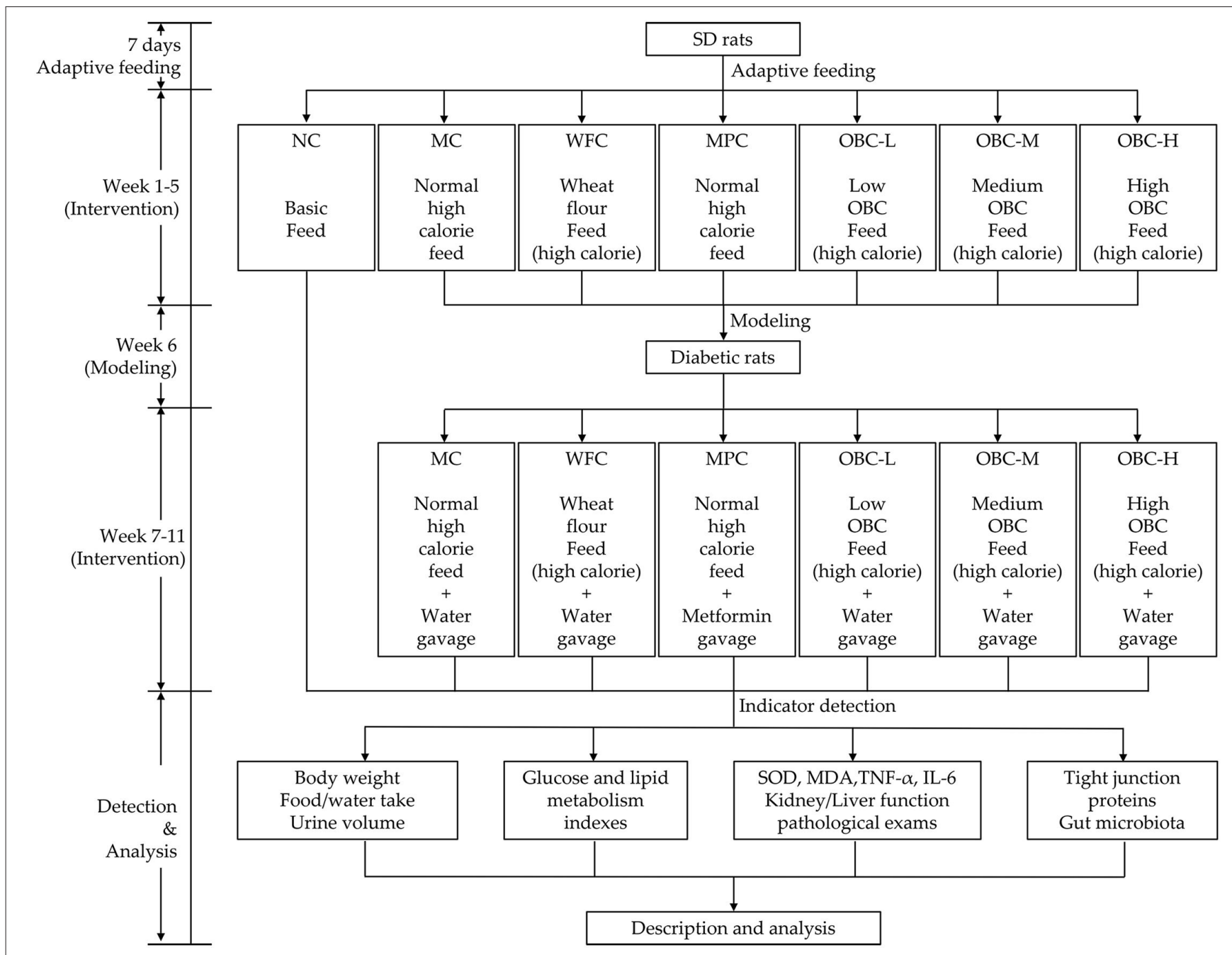

FIGURE 1 | Schematic diagram of the study protocol.

\section{Statistical Analysis}

Data accorded with normal distribution and homogeneity of variance were expressed as means $\pm \mathrm{SD}$, compared by oneway analysis of variance (ANOVA), otherwise, expressed as medians and quartiles, and compared by Kruskal-Wallis $\mathrm{H}$ test. SPSS 22.0 (IBM Corp, Armonk, NY, USA) was applied for statistical analysis. The value $p<0.05$ was considered statistically significant.

\section{RESULTS}

\section{Effect of OBC on General State and Bodyweight}

With withered yellow fur and less well-formed feces, the diabetic rats showed a much poorer haircoat quality and a symptom of diarrhea, compared with normal rats. Besides, rats with STZinduced diabetes also shared reduced general activity. As shown in Figure 2A, there were no significant differences among the seven groups of body weight before modeling. After the injection of STZ, compared with the normal group, the body weight of rats in MC, MPC, WFC, and OBC-M groups significantly decreased from the 7th week, whereas the OBC-H group decreased later from the 8 th week and the OBC-L group decreased from the 9th week. Compared with the MC group, the bodyweight of the OBC-L group was found markedly lower than that of the MC group from the 8 th week and the $\mathrm{OBC}-\mathrm{H}$ group in the 10 th week. From the 7th week to the 9th week, the bodyweight of the OBC$\mathrm{L}$ and $\mathrm{OBC}-\mathrm{H}$ groups was significantly higher than that of the WFC group.

As shown in Figure 2B, compared with the normal group, water intake was found obviously increased in the MC and WFC groups from the 8th week. Water intake in the OBC-L and OBC$\mathrm{M}$ groups was markedly lower than that in the MC and WFC groups from the 9th week. Food intake made no difference. This result suggests that $\mathrm{OBC}$ could improve diabetic symptoms in diabetic rats. 

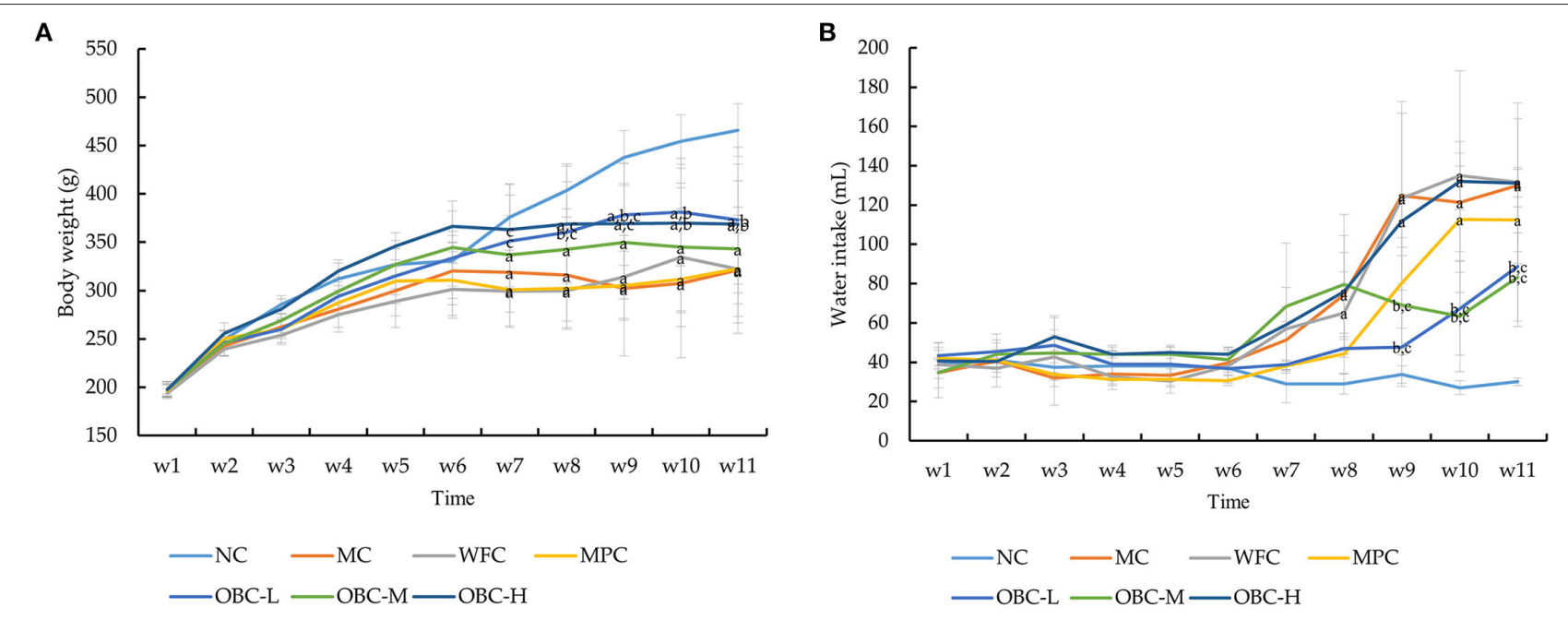

FIGURE 2 | Effect of OBC on body weight (A), water intake (B). NC $(n=10), \operatorname{MC}(n=9)$, WFC $(n=9)$, MPC $(n=8)$, OBC-L $(n=8)$, OBC-M $(n=10)$, OBC-H $(n=9)$, values are presented as mean \pm SD. (a) $p<0.05$ vs. NC rats, (b) $p<0.05$ vs. MC rats, (c) $p<0.05$ vs. WFC rats.

\section{Effect of OBC on Glucose Metabolism}

The fasting blood glucose of all rats showed a normal level $(6.3 \pm 0.91 \mathrm{mmol} / \mathrm{L})$, and no significant differences were seen among these groups before modeling. After the diabetic model was established, different from the normal group, the FBG level statistically increased over time in the diabetic rats since the 7th week (Figure 3A). By the end of the treatment by $\mathrm{OBC}$, rats in the OBC-L and OBC-M groups showed a significantly lower FBG level than rats in the WFC and MC groups (Figure 3B). This suggests that $\mathrm{OBC}$ could reduce fasting blood glucose in diabetic rats.

To analyze the effect of $\mathrm{OBC}$ on glucose tolerance in diabetic rats, we conducted the OGTT in the third, 7th, and 11th weeks. As shown in Table 3, there were no significant differences in OGTT-AUC among these groups before modeling, and after that, OGTT-AUC was found obviously increased in all diabetic rats in the 7th week. By the end of the experiment, rats treated with OBC showed some decreases of OGTT-AUC, among which, rats in the OBC-L group decreased statistically. These results suggest that $\mathrm{OBC}$ treatment may be conducive for the improvement of glucose tolerance in diabetic rats.

As shown in Figure 3C, compared with normal rats, the insulin level in the MC group was found obviously increased. After the intervention of metformin and $\mathrm{OBC}$, rats in MPC and $\mathrm{OBC}$ groups showed a significantly lower insulin level. The HOMA-IR index of all diabetic rats is higher than that of normal rats significantly, suggesting that insulin resistance in diabetic rats had occurred. After the treatment of wheat flour, metformin, and $\mathrm{OBC}$, compared with the MC group, the HOMA-IR index of WFC, MPC, and OBC groups were all dramatically decreased by the end of the experiment. Meanwhile, diabetic rats treated with OBC showed a lower HOMA-IR index than rats treated with wheat flour markedly (Figure 3D).

\section{Effect of OBC on Blood Lipid Metabolism}

As shown in Figure 4, the blood TC and LDL-C concentration of diabetic rats were significantly higher than that of normal rats, whereas the concentration of HDL-C was significantly lower. With the intervention of $\mathrm{OBC}$, serum TC levels of rats in $\mathrm{OBC}-\mathrm{M}$ and $\mathrm{OBC}-\mathrm{H}$ groups were significantly lower than those in diabetic rats, serum HDL-C in all OBC groups increased significantly, and LDL-C decreased significantly, indicating that $\mathrm{OBC}$ could improve lipid metabolism. No significant differences showed in the TG level among these groups.

\section{Effect of OBC on Serum SOD and MDA}

The serum SOD level was significantly lower in MC and WFC groups as compared with normal rats; meanwhile, diabetic rats in OBC groups showed a markedly higher SOD level than rats in the MC group (Figure 5A), showing that $\mathrm{OBC}$ treatment could improve diabetic rat's antioxidant capacity. No significant differences were shown in the MDA level among these groups (Figure 5B).

\section{Effect of OBC on Serum TNF- $\alpha$ and IL-6}

As shown in Figure 6, compared with the NC group, diabetic rats in the MC group showed increased serum TNF- $\alpha$ and IL6. Rats in the WFC group and rats that intervened with OBC showed reduced serum TNF- $\alpha$. The concentration of IL- 6 in OBC groups was significantly lower than that in the MC group but IL-6 in the OBC-L group was statistically lower than the WFC group. Compared with rats interfered with metformin, the level of IL- 6 in all OBC groups decreased, and TNF- $\alpha$ in the OBC-M group decreased. 


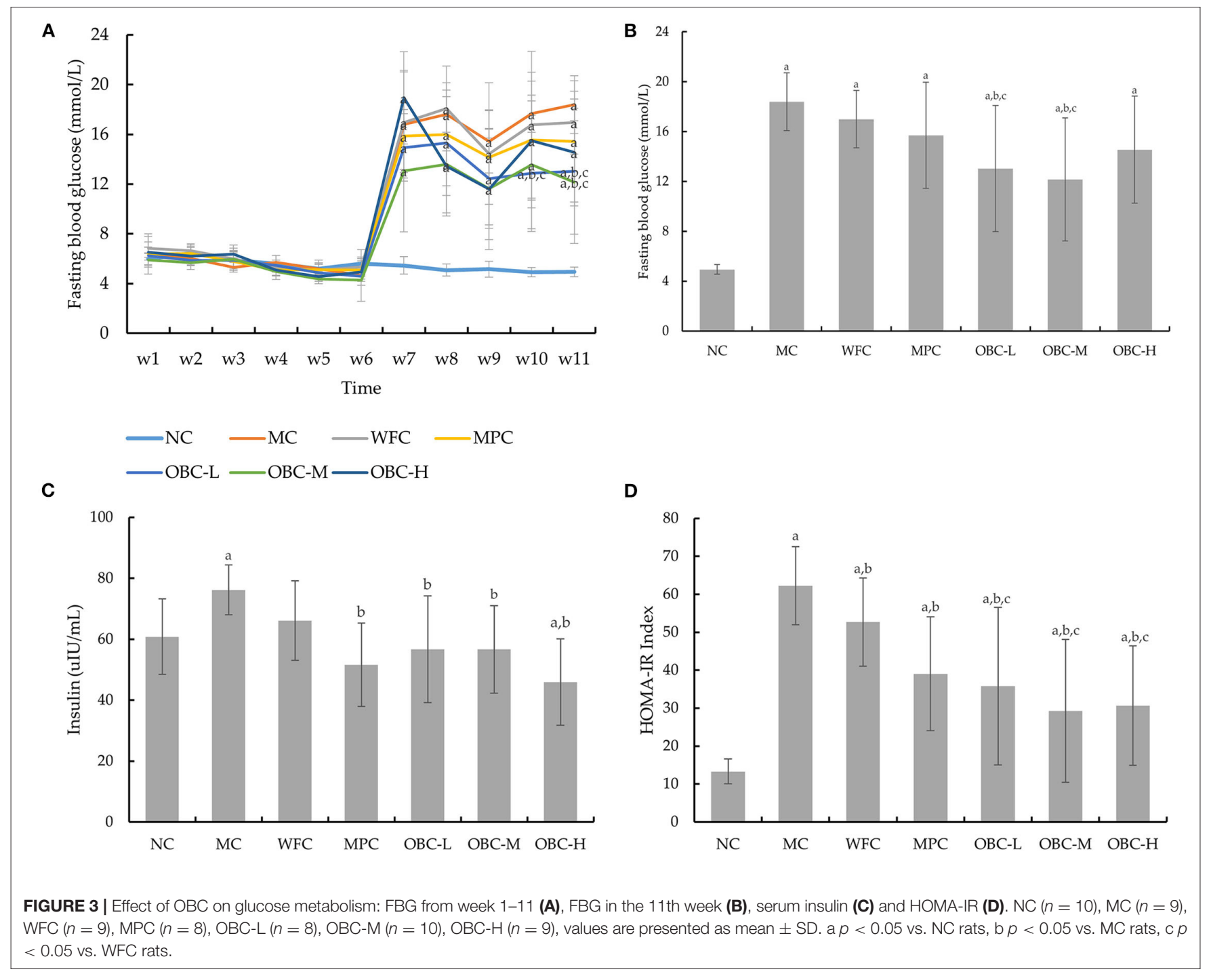

TABLE 3 | Effect of OBC on OGTT-AUC.

\begin{tabular}{lccc}
\hline Group & \multicolumn{2}{c}{ OGTT-AUC (mmol/L) } \\
\cline { 2 - 4 } & 3rd week & 7th week & 11th week \\
\hline NC & $13.10 \pm 0.75$ & $12.68 \pm 1.01$ & $12.17 \pm 0.75$ \\
MC & $13.72 \pm 0.98$ & $45.60 \pm 6.78^{\mathrm{a}}$ & $46.44 \pm 10.52^{\mathrm{a}}$ \\
WFC & $13.62 \pm 1.09$ & $48.29 \pm 6.95^{\mathrm{a}}$ & $47.91 \pm 7.77^{\mathrm{a}}$ \\
MPC & $13.50 \pm 1.46$ & $42.76 \pm 7.36^{\mathrm{a}}$ & $46.42 \pm 9.83^{\mathrm{a}}$ \\
OBC-L & $13.20 \pm 1.29$ & $36.61 \pm 14.70^{\mathrm{a}}$ & $28.74 \pm 13.56^{\mathrm{a}, \mathrm{b}, \mathrm{c}}$ \\
OBC-M & $13.16 \pm 0.98$ & $43.17 \pm 10.21^{\mathrm{a}}$ & $41.38 \pm 11.74^{\mathrm{a}}$ \\
OBC-H & $14.05 \pm 0.73$ & $49.49 \pm 5.92^{\mathrm{a}}$ & $43.87 \pm 11.99^{\mathrm{a}}$ \\
\hline
\end{tabular}

NC, normal control group; MC, model control group; WFC, wheat flour control group; MPC, metformin positive control group; OBC-L, low-dose group; OBC-M, medium-dose group; $O B C-H$, high-dose group. Values are presented as mean $\pm S D$. ${ }^{a} P<0.05$ vs. NC rats, ${ }^{b} P<0.05$ vs. MC rats, ${ }^{c} P<0.05$ vs. WFC rats.

\section{Effect of OBC on Liver Function}

Serum ALT and AST levels can reflect the degree of liver cell injury. The ALT data, which does not conform to the normal distribution, is represented by a boxplot and analyzed by
Kruskal-Wallis $\mathrm{H}$ tests, showing that compared with rats in the NC group, serum ALT in diabetic rats increased significantly, and the ALT levels of rats in OBC groups were significantly lower than rats in the MC, WFC, and MPC groups (Figure 7A). The serum 


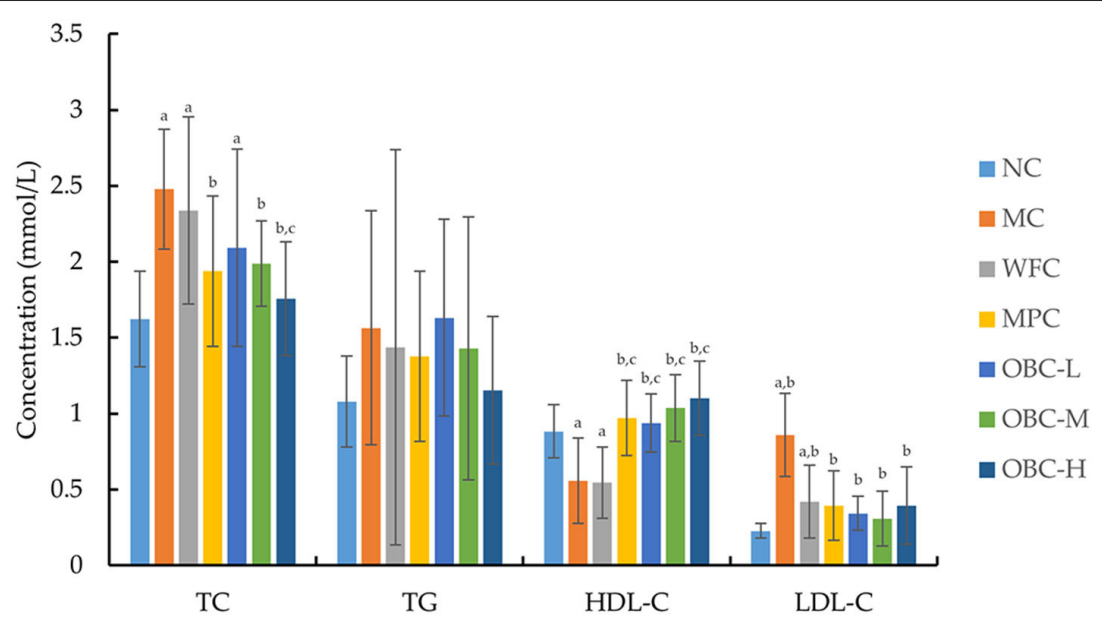

FIGURE 4 | Effect of OBC on blood lipid metabolism. NC $(n=10), \operatorname{MC}(n=9), \operatorname{WFC}(n=9), \operatorname{MPC}(n=8), \mathrm{OBC}-\mathrm{L}(n=8), \mathrm{OBC}-\mathrm{M}(n=10), \mathrm{OBC}-\mathrm{H}(n=9)$, values are presented as mean $\pm \mathrm{SD}$. a $p<0.05$ vs. NC rats, $\mathrm{b} p<0.05$ vs. MC rats, $\mathrm{c} p<0.05$ vs. WFC rats.
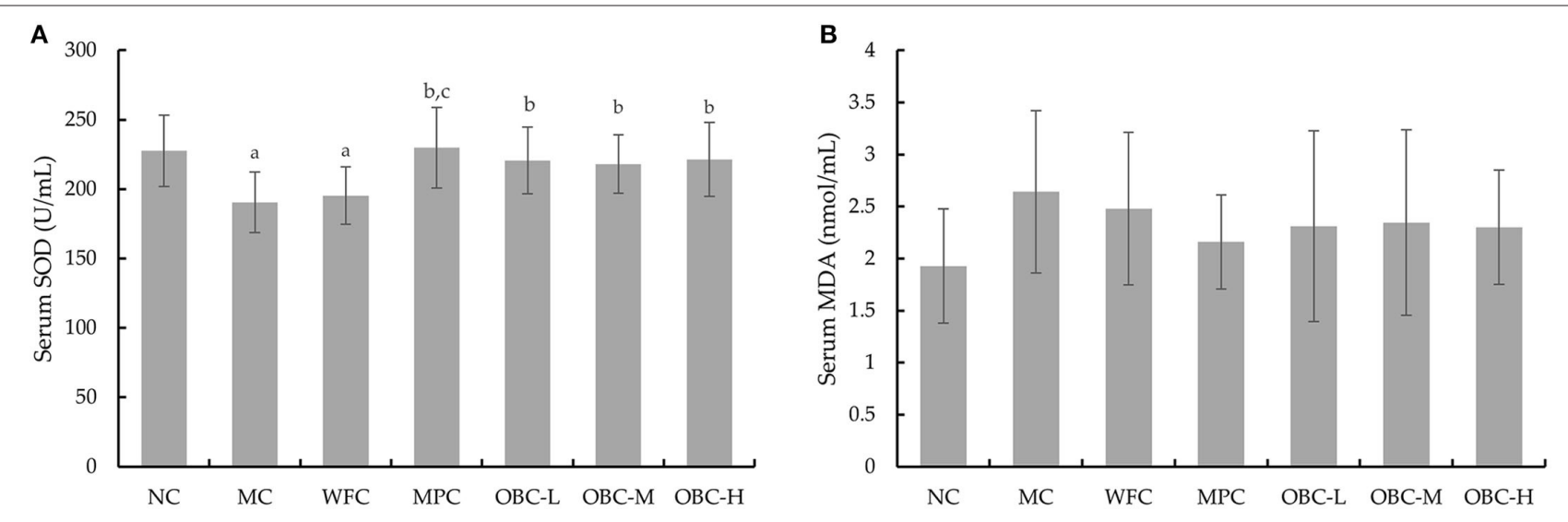

FIGURE 5 | Effect of OBC on SOD (A) and MDA (B).NC ( $n=10)$, MC $(n=9)$, WFC $(n=9), \operatorname{MPC}(n=8), \mathrm{OBC}-\mathrm{L}(n=8), \mathrm{OBC}-\mathrm{M}(n=10)$, OBC-H $(n=9)$, values are presented as mean \pm SD. a $p<0.05$ vs. NC rats, b $p<0.05$ vs. MC rats, $c p<0.05$ vs. WFC rats.
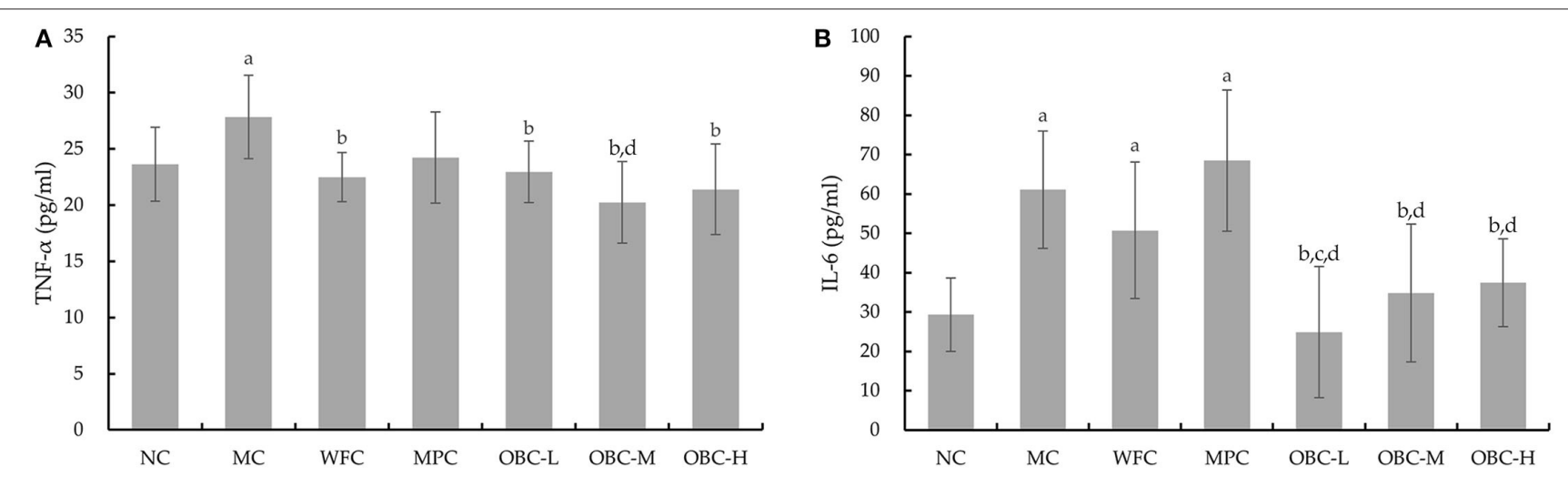

FIGURE 6 | Effect of OBC on TNF- $\alpha$ (A) and IL-6 (B).NC $(n=10), \mathrm{MC}(n=9)$, WFC $(n=9)$, MPC $(n=8), \mathrm{OBC}-\mathrm{L}(n=8), \mathrm{OBC}-\mathrm{M}(n=10), \mathrm{OBC}-\mathrm{H}(n=9)$, values are presented as mean $\pm \mathrm{SD}$. a $p<0.05$ vs. NC rats, $\mathrm{b} p<0.05$ vs. MC rats, $\mathrm{c} p<0.05$ vs. WFC rats, $\mathrm{d} p<0.05$ vs. MPC rats. 


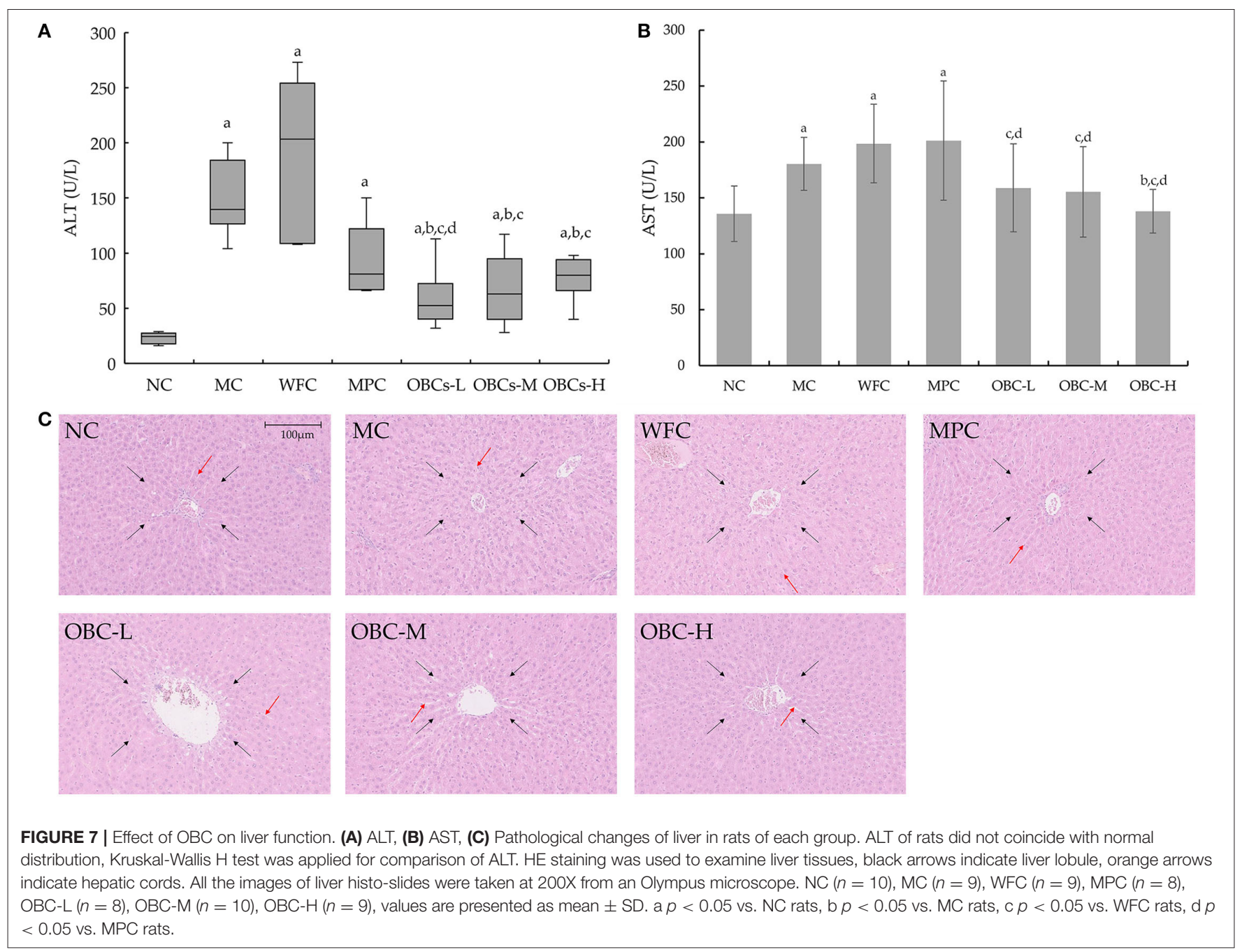

AST of rats in MC, WFC, and MPC groups were significantly higher than that of normal rats. The AST of rats in the OBC groups were significantly lower than that of the WFC group, whereas the AST of the OBC-H group was significantly lower than that of the MC group (Figure 7B). As shown in Figure 7C, rats in the NC group had the normal structure of hepatocytes with regular radical morphology of hepatic architecture with normal sinusoids. Diabetic rats in MC and WFC groups showed hepatocellular hypertrophy with disordered structure of liver lobule, narrowed and unrecognizable hepatic cords. By contrast, rats intervened with metformin and $\mathrm{OBC}$ showed regular liver cells and improved liver lobules and cords. Compared with rats interfered with metformin, the level of AST in all OBC groups decreased, and ALT in the OBC-l group decreased.

\section{Effect of OBC on Kidney Function}

All diabetic rats showed a classic symptom of polyuria, as shown in Figure 8A. The 24-h urine volume of all diabetic rats was significantly higher than that of the rats in the NC group, and with the treatment of $\mathrm{OBC}$, the 24-h urine volume of rats in the OBC-M group was markedly reduced, compared with rats in MC and WFC groups. Likewise, all diabetic rats showed an obviously increased kidney coefficient than normal rats, whereas rats in the OBC-L group showed a significantly decreased kidney coefficient compared with that in MC and WFC groups (Figure 8B).

As shown in Figure 8C, using HE staining, we found there were increases in glomerular volume and decreases of the renal capsule in renal tissues of MC, WFC, and MPC groups; in contrast, renal tissues in $\mathrm{OBC}$ groups were normal. Meanwhile, swelled renal tubular epithelial cells had been observed in MC and WFC groups, whereas less pathological changes showed in MPC and OBC groups. As shown in Figure 8D, compared with normal rats in the NC group, diabetic rats in the MC group showed increased glomerular volume, thickened capillary basement membrane, and vacuolar degeneration of renal tubular epithelial cells, indicating that significant renal tissue damage occurred in diabetic rats. Similar pathological damages occurred in rats in the WFC group. After the intervention of OBC, the renal damage of rats in each OBC group was improved. The structure of the glomerular basement membrane of rats intervened with $\mathrm{OBC}$ was clear and complete, and the 

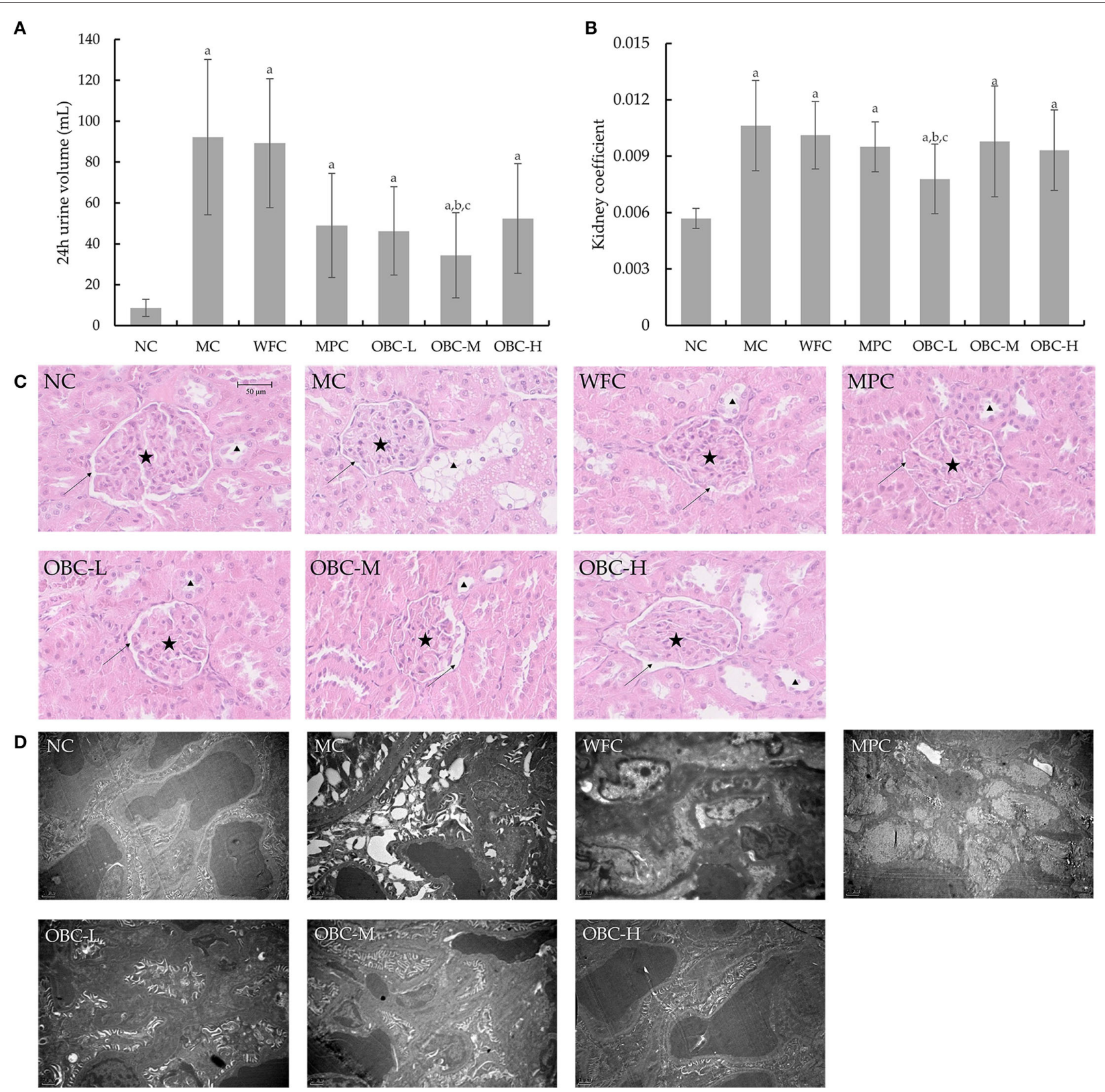

FIGURE 8 | Effect of OBC on kidney function. (A) Six rats in each group were randomly selected to collect their $24 \mathrm{~h}$ urine at the end of the experiment. (B) The kidney coefficient is the ratio of kidney weight (g) to body weight (g). (C) HE staining was used to examining renal tissues, arrows indicate renal capsules, pentacles indicate glomeruli of kidneys and triangles indicate renal tubular epithelial cells. (D) Ultra microstructural changes of kidney in rats. NC $(n=10)$, MC $(n=9)$, WFC $(n=$ 9), MPC ( $n=8)$, OBC-L $(n=8), \mathrm{OBC}-\mathrm{M}(n=10), \mathrm{OBC}-\mathrm{H}(n=9)$, values are presented as mean $\pm \mathrm{SD}$. a $p<0.05$ vs. NC rats, b $p<0.05$ vs. MC rats, c $p<0.05$ vs. WFC rats.

arrangement of vascular endothelial cells was regular without obvious abnormalities.

\section{Effect of OBC on lleal Intestinal Barrier}

Compared with normal rats, the expression of Claudin-1, ZO1 , and Occludin was weakened significantly in diabetic rats in the MC group (Figure 9). All the rats that intervened with OBC showed enhanced expression of Claudin-1 and ZO-1, with the expression of Occludin enhanced in the OBC-M group. Besides, the expression of $\mathrm{ZO}-1$ in the $\mathrm{OBC}-\mathrm{M}$ and $\mathrm{OBC}-\mathrm{H}$ groups was higher than that in the WFC group. Rats in the OBC-M and OBC-H groups showed higher ZO-1 expression than that of the MPC group. The morphology and ultrastructure of ileal villi in the NC group were normal, and no gap widening was observed. 


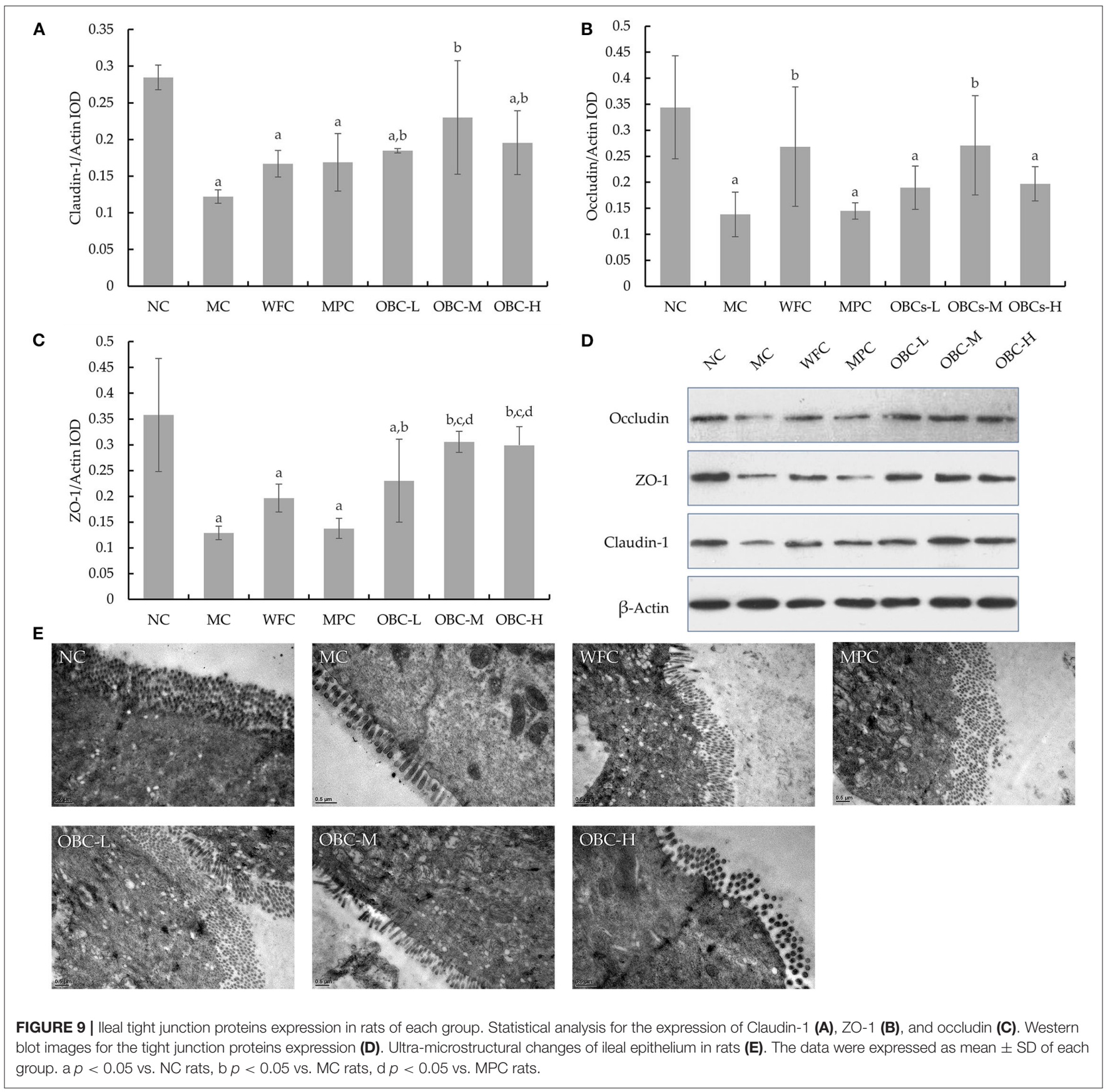

However, the ileal villi of diabetic rats were swollen, and the celltight junction structure was disordered. The ileal epithelial cell morphology and cell tight junction devices of rats treated with WFC and OBC were improved.

\section{Effect of OBC on Gut Microbiota Operational Taxonomic Units}

According to the sequence similarity (>97\%), high-quality sequences were classified into multiple operational taxonomic units (OTUs) using QIIME to facilitate analysis. The number of OTUs detected in the cecal stool samples of rats in the seven test groups (NC, MC, WFC, MPC, OBC-L, OBC-M, OBC-H) were $507,542,562,505,516,524,522$, respectively, whereas the number of unique OTUs was $6,4,12,1,2,6,6$, respectively. There were 331 OTUs shared by seven groups.

\section{Alpha Diversity Analysis}

The observed species can reflect the actual number of OTUs observed. Chaol is an estimator of phylotype richness, and the Simpson index of diversity reflects both the richness and community evenness. In this study, there was no significant difference found in the observed species $(p=0.26)$ and Chaol 

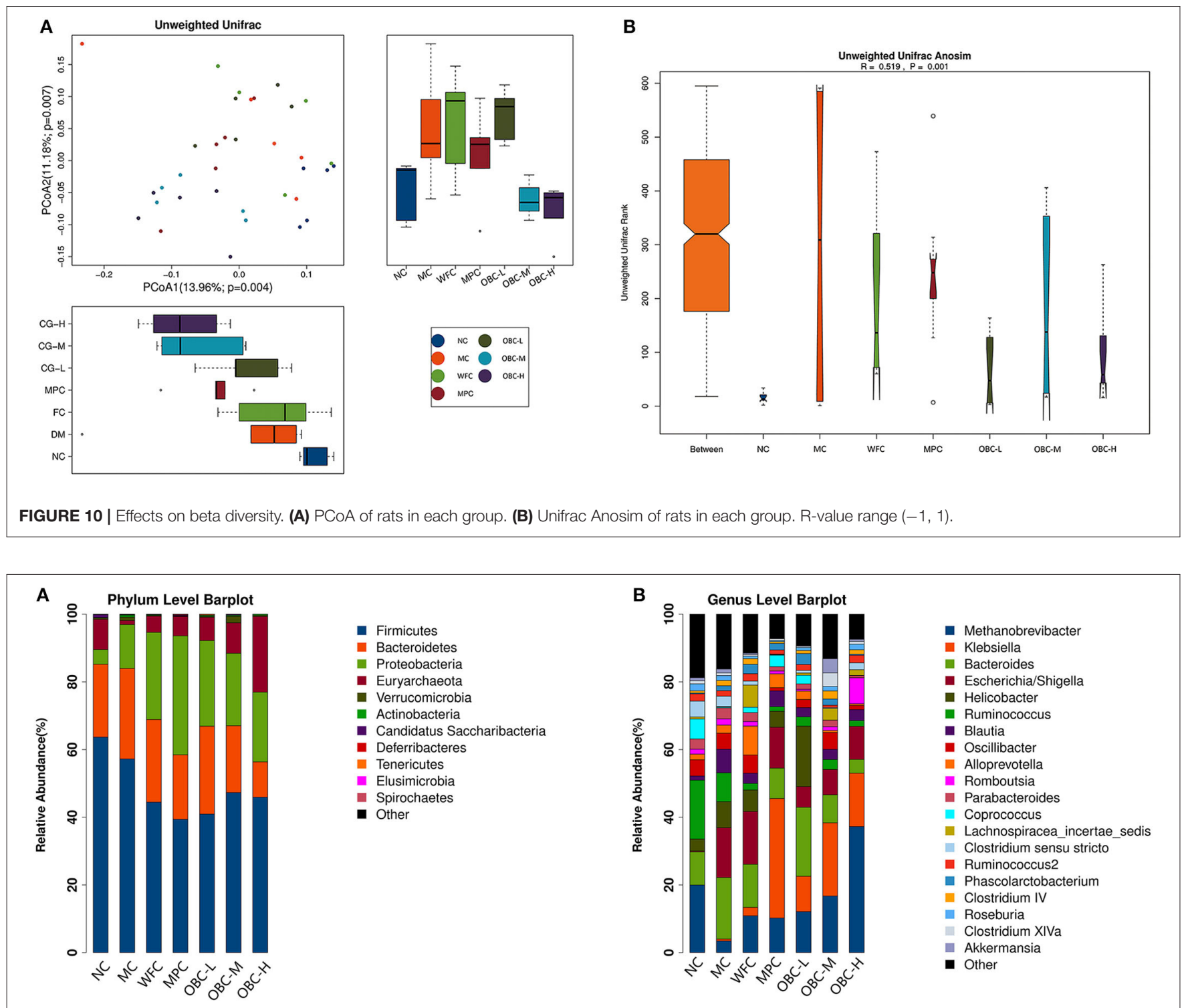

FIGURE 11 | Microbial distributions at the phylum level (A) and the genus level (B) in the fecal samples from the seven groups. Each bar represents the microbiota composition of one group.

index $(p=0.49)$, but there was a significant difference in the Simpson index $(p=0.02)$. MC group had higher evenness indexes $[0.97(0.98,0.96)]$ than the NC group $[0.96(0.99,0.93)]$, whereas each intervention group had a lower evenness index than MC group, WFC: 0.95 (0.97, 0.92), MPC: 0.91 (0.98, 0.84), OBCL: 0.95 (0.98, 0.91), OBC-M: 0.93 (1.02, 0.85), OBC-H: 0.91 (0.99, 0.82 ). Indicators of alpha diversity were reported as the median $\pm \mathrm{IQR}$.

\section{Beta Diversity Analysis}

Beta diversity analysis represents the extent of similarity between different microbial communities. Two principal components were extracted by PCoA. Figure 10 showed a clear separation between the fecal samples from the MC group and NC group, whereas OBC-M and OBC-H groups were close with the $\mathrm{NC}$ group. Percentage values at the axes indicated the contribution of the principal components to the explanation of total variance in the dataset. The figure showed that the percentages of variation explained by PC1 and PC2 were 13.96 and $11.18 \%$, respectively.

In addition, ANOSIM demonstrated the differences in the gut microbiota among these groups. The intergroup differences among these groups were greater than the intragroup differences $(R=0.519, p=0.001)$.

\section{Classification Abundance Analysis}

Figure 11 showed microbial distributions at the phylum and genus level in the fecal samples from the seven groups. Firmicutes and Bacteroidetes accounted for the largest proportion at the 
phylum level. At the genus level, Methanobrevibacter was the most proportional.

\section{Axonomic Composition}

To explore the specific bacterial taxa associated with $\mathrm{OBC}$ treatment, a LEfSe comparison of the gut microbiota among these seven groups was performed. The greatest difference in taxa from phylum to genus level was identified via the LDA score. Compared with normal rats, the Firmicutes phyla of diabetic rats is significantly reduced and the Actinobacteria phyla increased.

There were four significantly different phyla, with enrichment of Firmicutes in NC group, Actinobacteria in MC group, Proteobacteria in MPC group, and Euryarchaeota in OBC-H group.

Bacterial genus including Clostridium sensu stricto, Sutterella, Saccharibacteria genera incertae sedis, Clostridium XlVb, and Paraprevotella were found enriched in the NC group, Facklamia, Prevotella, Rhodococcus, Terrisporobacter, and Subdivision 5 genera incertae sedis were found enriched in the MC group, and Escherichia_Shigella, Oscillibacter, Flavonifractor, and Weissella were found enriched in the WFC group, Klebsiella was found enriched in MPC group. Helicobacter, Phascolarctobacterium, and Parasutterella were significantly intensified in the OBC-L group, Clostridium IV and Brevundimonas were significantly intensified in the OBC-M group and Methanobrevibacter, Methanosphaera, Enterococcus, Turicibacter, Eubacterium, and Anaerotruncus significantly intensified in the $\mathrm{OBC}-\mathrm{H}$ group.

\section{DISCUSSION}

In this work, we demonstrated that as a staple food substitute, OBC has health-promoting effects on diabetes. To clarify these effects, we followed the changes in general state, blood glucose, and glucose tolerance of diabetic rats during the intervention of OBC and detected certain blood biomarkers. Diabetic rats intervened by $\mathrm{OBC}$ showed improved states, including increased body weight, decreased water take, and decreased urine volume. With decreased FBG, OGTT-AUC level as well as serum insulin level and HOMA-IR, rats in OBC groups showed improved glycometabolism and insulin resistance. Meliorated liver and kidney functions and antioxidant levels also showed that $\mathrm{OBC}$ can improve antioxidant capacity and reduce organ damage caused by diabetes. What is more, by promoting the expression of tight junction proteins in the intestine, $\mathrm{OBC}$ can improve the intestinal barrier. In the meantime, the consumption of $\mathrm{OBC}$ can increase the abundance of intestinal microbes and improve gut microbiota disturbance.

Diabetes has several common symptoms including polydipsia, polyuria, polyphagia, hyperglycemia, fatigue, and weight loss (14). STZ can cause pancreatic islet $\beta$-cell destruction, and is widely used experimentally to produce a diabetic rat model $(10,15,16)$. In this work, after the injection of STZ at the end of the 6th week, while the FBG of the normal group rats remained in a normal range $(5.45 \pm 0.70 \mathrm{mmol} / \mathrm{L})$, rats in each group injected by STZ showed significantly increased FBG, which ranged from $13.07 \pm 4.92 \mathrm{mmol} / \mathrm{L}$ to $18.94 \pm 3.71 \mathrm{mmol} / \mathrm{L}$, indicating that the diabetic rat model was successfully established, which is consistent with previous studies on diabetic animal models (10). Besides, different from the continuous increase in body weight of the normal group, the weight of diabetic rats no longer gained or tend to decrease. The water consumption of diabetic rats continued to increase, as a symptom of polydipsia. Diabetic rats failed to keep a clean haircoat and showed a sign of fatigue. Elevated OGTT-AUC reflects the decrease of glucose tolerance in diabetic rats, the increase of HOMA-IR shows the formation of insulin resistance, with the raised FBG and insulin level, disorder of glucose metabolism in diabetic rats revealed. As shown in Table 3, the increased OGTT-AUC level of rats in the OBC-L group suggests that the intervention of $\mathrm{OBC}$ may be conducive to improve glucose tolerance in diabetic rats, which is in accordance with previous reports $(10,17)$. Relevantly, as shown in Figure 4, the consumption of $\mathrm{OBC}$ also led to an improvement in insulin metabolism and insulin resistance in diabetic rats, which in turn caused an improvement in glucose metabolism. Hyperglycemia-induced glycated molecules lead to increased oxidative stress in diabetic rats, which further resulted in the aggravation of multiple diabetic complications, including renal tubular injury $(18,19)$. In our study, diabetic rats intervened with OBC showed significantly reduced 24-h urine volume and kidney coefficient with ameliorated renal tissues and ultramicrostructure, whereas the serum SOD level markedly increased, indicating that $\mathrm{OBC}$ could reduce kidney injuries by enhancing antioxidant capacity. Our data also suggested that OBC may improve glucose metabolism by reducing oxidative stress. In previous studies, few of them had focused on the effect of interventions to reduce urine volume in diabetic rats, and our study showed that $\mathrm{OBC}$ could successfully reduce the urine volume of diabetic rats in $24 \mathrm{~h}$, while reducing damages to the kidney caused by diabetes. Along with the result that rats in OBC groups showed less withered haircoat and less fatigue, these results showed an improvement in the quality of life of diabetic rats treated with $\mathrm{OBC}$.

Several studies $(8,20-24)$ in relevant fields had demonstrated that oat oligopeptides, oat $\beta$-glucan, and buckwheat iminosugar d-fagomine could exert a hypoglycemic effect. Likewise, we tested the FBG of rats in each group weekly and did the oral glucose tolerance test biweekly, and the results confirmed that FBG and OGTT-AUC in OBC groups were lower than diabetic model rats and rats treated with wheat flour. Unlike our work, a previous experiment (10) showed that oat oligopeptides failed to cause changes in insulin in diabetic rats, and could improve insulin resistance and SOD level only if the oat oligopeptides were given in a high dose $(2.00 \mathrm{~g} / \mathrm{kg}$ body weight), though it may reduce the glucose level in diabetic rats. What's more, by contrast, in our work, after the kidney coefficient of diabetic rats increased, the intervention of $\mathrm{OBC}$ could reduce it. However, unlike the previous experiment, our work intervenes diabetic rats with the compound of two whole grains instead of a single ingredient of oat. Other functional ingredients of oat and buckwheat may be involved, considering the high level of rutin, quercetin, proteins, and minerals in buckwheat (20) and the high content of $\beta$-glucose and dietary fiber in oat, many of which have been proved to improve diabetes. In addition, our intervention of $\mathrm{OBC}$ had been implemented since the beginning of the experiment rather than 
after the injection of STZ. These differences in the intervention may account for the different results. However, while indicators of glucose metabolism such as FBG, OGTT-AUC and insulin, and proinflammatory cytokines, such as IL-6, in diabetic rats intervened with different doses of $\mathrm{OBC}$ were improved compared with rats in the $\mathrm{MC}$ group, suggesting that the intervention of OBC may correlate with the improvement of glucose metabolism and inflammatory reaction in diabetes, the phenotypes of each intervention group are not consistent throughout the work, and not all differences between the intervention groups and the MC group are statistically significant $(p<0.05)$. Thus, there was no clear dose-response relationship between $\mathrm{OBC}$ intervention and improvement of diabetes. This may be due to the complexity of ingredients in OBC and intervention pathways for diabetes. In addition, the limited sample size and grouping dose level in this work may also preclude the discovery of the dose-response relationship. Follow-up studies can explore a clearer dose-effect relationship by increasing the sample size and reducing the dosage gradient.

There is growing evidence that gut microbiota is closely associated with metabolic diseases including type 2 diabetes (2528). Specific changes in the diversity of gut microbiota are one of the characteristics of diabetic patients and animals, and diet is one of the most influential factors that impacts the dynamic microbial community ecosystem (29). In the current work, the results from the $16 \mathrm{~s}$ rDNA assay showed that $\mathrm{OBC}$ had a significant effect on gut microbiota in diabetic rats. While the Simpson index was increased in the MC group, diabetic rats treated with $\mathrm{OBC}$ showed a lower Simpson index, indicating that OBC treatment could make gut microbiota show a lower evenness. What's more, Beta diversity analysis showed that the gut microbial community of MC rats was significantly different from that of normal rats, whereas the gut microbial community of OBC-M and OBC$\mathrm{H}$ rats was more similar to that of normal rats, indicating that $\mathrm{OBC}$ may exert to alter microbiota composition to make it more resembling of microbiotas of healthy rats.

Reasons for OBC impacting gut microbiota may be related to its components. Higher Parasutterella abundance was observed in the OBC-L group. Though there is insufficient knowledge on the relationship between specific strains and dietary components, evidence has shown that complex carbohydrates may be involved. Yuan et al. (30) found that polysaccharides could increase the abundance of Parasutterella. Fermented by gut bacteria, various complex carbohydrates could be the major fuel source of the microbiota $(31,32)$ to exert a major impact to promote the growth of the probiotic and positively influence probioticenterocyte interaction (33). Besides, unique phytochemicals contained in $\mathrm{OBC}$, such as buckwheat phenolic acids, ferulic acid, and oat avenanthramides, have been shown to favor specific bacterial species (32-34).

The underlying mechanisms of gut microbiota impacted by $\mathrm{OBC}$ regulating host metabolism may include favorable changes in bacterial composition and/or activity, improvement of intestinal barrier function (35), productions of short-chain fatty acids (SCFAs), and inhibition of inflammation $(36,37)$. Certain species of probiotics like lactobacilli possess potent alphaglucosidase inhibitory activity that prevents the breakdown of complex carbohydrates and reduces postprandial hyperglycemia (38), while other bacteria like Firmicutes are usually involved in the transport of nutrients and facilitates the absorption and fermentation of SCFA, which are essential for maintaining insulin sensitivity (39) and reducing local inflammation by regulating the expression of proinflammatory cytokines as TNF$\alpha$ and IL- 6 through the activation of macrophages and dendritic cells (40). TNF- $\alpha$ and IL- 6 are important inflammatory factors involved in inflammatory response, often used as diagnostic indicators of diabetes and related complications such as diabetic nephropathy. On the contrary, potentially pathogenic bacteria as Rhodococcus could inhibit the expression of tight junction proteins like occludin, claudin-1, and ZO-1, while it produces metabolites that can destroy the epithelial barrier and increase gut permeability $(38,41)$. Thus, microbiota products such as lipopolysaccharide can drive low-grade inflammation, which had long been recognized as a potential cause of insulin resistance (36, 42). However, unlike the commonly reported findings $(32,43)$, no intergroup difference was found in bifidobacteria and lactobacilli in this study. This may be due to the strain of experimental animals, feeding diets, and geographical location (44).

In addition, this study shows that $\mathrm{OBC}$ can reduce inflammation, moderate liver damage, and improve the intestinal barrier, whereas metformin, which is a commonly used drug for diabetes treatment, cannot. It is noteworthy that there was no difference in food taken, this suggesting the palatability of OBC. Besides, compared with medication, people are also more receptive to $\mathrm{OBC}$, common foods in daily life, and thus $\mathrm{OBC}$ is a more suitable potential food for diabetes prevention and long-term intervention.

Although this work could provide new insights to explore the diet intervention on diabetes, it has several limitations. First, in our work there was only one compounding ratio of buckwheat and oat, and more compounding ratios may enhance the experimental evidence. Second, although this work shows that the consumption of $\mathrm{OBC}$ is closely related to the improvement of diabetes and gut microbiota ameliorating, further research, such as fecal bacteria transplantation, is needed to decipher the causal relationship involving bacterial disequilibrium and diabetes and to explore key bacteria which may play an important role in the intervention. Third, follow-up studies are needed to support the improvement of $\mathrm{OBC}$ on glucose tolerance in diabetes, and to explore the recommended dose of $\mathrm{OBC}$ for the specific dietary pattern.

\section{CONCLUSION}

The results revealed that as a staple food substitute, OBC could exert promoting effects on diabetic rats. By improving antioxidant capacity, reducing inflammation, and improving the imbalance of intestinal microbiota, the glucose and lipid metabolism and insulin resistance of diabetic rats have been improved. Meanwhile, by improving a variety of tissues and organs in the diabetic state, $\mathrm{OBC}$ may have the potential to prevent diabetic complications. OBC can be used as a potential 
staple food substitute in dietary patterns that are suitable for the Chinese.

\section{DATA AVAILABILITY STATEMENT}

The original contributions presented in the study are included in the article/Supplementary Material, further inquiries can be directed to the corresponding author.

\section{ETHICS STATEMENT}

The animal study was reviewed and approved by Ethics Committee of Peking University.

\section{AUTHOR CONTRIBUTIONS}

SL and JW: conceptualization and methodology. SL: formal analysis and writing original draft preparation. SL, CH, XL, $\mathrm{XY}, \mathrm{HM}$, and $\mathrm{XZ}$ : investigation. YX: resources. SL and XY: data curation. MX and JW: writing review and editing. YL and JW: supervision. JW: project administration and funding

\section{REFERENCES}

1. Saeedi P, Petersohn I, Salpea P, Malanda B, Karuranga S, Unwin N, et al. Global and regional diabetes prevalence estimates for 2019 and projections for 2030 and 2045: Results from the International Diabetes Federation Diabetes Atlas, 9(th) edition. Diabetes Res Clin Pract. (2019) 157:107843. doi: 10.1016/j.diabres.2019.107843

2. Hou YY, Ojo O, Wang LL, Wang Q, Jiang Q, Shao XY, et al. A randomized controlled trial to compare the effect of peanuts and almonds on the cardiometabolic and inflammatory parameters in patients with type 2 diabetes mellitus. Nutrients. (2018) 10:1565. doi: 10.3390/nu10111565

3. Sakuma M, Yamanaka-Okumura H, Naniwa Y, Matsumoto D, Tsunematsu M, Yamamoto H, et al. Dose-dependent effects of barley cooked with white rice on postprandial glucose and desacyl ghrelin levels. JClin Biochem Nutr. (2009) 44:151-9. doi: 10.3164/jcbn.08-232

4. Perez-Ramirez IF, Becerril-Ocampo LJ, Reynoso-Camacho R, Herrera MD, Guzman-Maldonado SH, Cruz-Bravo RK. Cookies elaborated with oat and common bean flours improved serum markers in diabetic rats. J Sci Food Agric. (2018) 98:998-1007. doi: 10.1002/jsfa.8548

5. Boffetta P, Thies F, Kris-Etherton P. Epidemiological studies of oats consumption and risk of cancer and overall mortality. $\mathrm{Br}$ $J$ Nutr. (2014) 112 Suppl 2:S14-8. doi: 10.1017/S00071145140 02268

6. Qiu J, Li Z, Qin Y, Yue Y, Liu Y. Protective effect of tartary buckwheat on renal function in type 2 diabetics: a randomized controlled trial. Ther Clin Risk Manag. (2016) 12:1721-7. doi: 10.2147/TCRM.S123008

7. Li X, Cai X, Ma X, Jing L, Gu J, Bao L, et al. Short- and long-term effects of wholegrain oat intake on weight management and glucolipid metabolism in overweight type-2 diabetics: a randomized control trial. Nutrients. (2016) 8. doi: 10.3390/nu8090549

8. Qiu J, Liu Y, Yue Y, Qin Y, Li Z. Dietary tartary buckwheat intake attenuates insulin resistance and improves lipid profiles in patients with type 2 diabetes: a randomized controlled trial. Nutr Res. (2016) 36:1392401. doi: 10.1016/j.nutres.2016.11.007

9. Shen XL, Zhao T, Zhou Y, Shi X, Zou Y, Zhao G. Effect of oat betaglucan intake on glycaemic control and insulin sensitivity of diabetic patients: a meta-analysis of randomized controlled trials. Nutrients. (2016) 8:39. doi: 10.3390/nu8010039 acquisition. All authors contributed to the article and approved the submitted version.

\section{FUNDING}

This research was funded by the Ministry of Science and Technology of the People's Republic of China, Grant Number 2017YFD0401202.

\section{ACKNOWLEDGMENTS}

We are grateful to the College of Food Science and Nutritional Engineering, China Agricultural University for the nutrient detection of oat and buckwheat, and thankful to Lan Wu for her help in this study and Fangfang Ni for the animal experiments.

\section{SUPPLEMENTARY MATERIAL}

The Supplementary Material for this article can be found online at: https://www.frontiersin.org/articles/10.3389/fnut.2021. 762277/full\#supplementary-material
10. Wang JB, Liu XR, Liu SQ, Mao RX, Hou C, Zhu N, et al. Hypoglycemic effects of oat oligopeptides in high-calorie diet/STZ-induced diabetic rats. Molecules. (2019) 24:558. doi: 10.3390/molecules 24030558

11. Zhang B, Gao C, Li Y, Wang M. D-chiro-inositol enriched Fagopyrum tataricum (L) Gaench extract alleviates mitochondrial malfunction and inhibits ER stress/JNK associated inflammation in the endothelium. $J$ Ethnopharmacol. (2018) 214:83-9. doi: 10.1016/j.jep.2017.12.002

12. Lee DG, Jang IS, Yang KE, Yoon SJ, Baek S, Lee JY, et al. Effect of rutin from tartary buckwheat sprout on serum glucose-lowering in animal model of type 2 diabetes. Acta Pharm. (2016) 66:297-302. doi: 10.1515/acph-2016-0021

13. Tosh SM, Bordenave N. Emerging science on benefits of whole grain oat and barley and their soluble dietary fibers for heart health, glycemic response, and gut microbiota. Nutr Rev. (2020) 78:13-20. doi: 10.1093/nutrit/nuz085

14. Wang-Fischer $\mathrm{Y}$, Garyantes $\mathrm{T}$. Improving the reliability and utility of streptozotocin-induced rat diabetic model. J Diabetes Res. (2018) 2018:8054073. doi: 10.1155/2018/8054073

15. Gheibi S, Kashfi K, Ghasemi A. A practical guide for induction of type2 diabetes in rat: Incorporating a high-fat diet and streptozotocin. Biomed Pharmacother. (2017) 95:605-13. doi: 10.1016/j.biopha.2017.08.098

16. Reed MJ, Meszaros K, Entes LJ, Claypool MD, Pinkett JG, Gadbois TM, et al. A new rat model of type 2 diabetes: the fat-fed, streptozotocin-treated rat. Metabolism. (2000) 49:1390-4. doi: 10.1053/meta.2000.17721

17. Eriksen AK, Brunius C, Mazidi M, Hellstrom PM, Riserus U, Iversen KN, et al. Effects of whole-grain wheat, rye, and lignan supplementation on cardiometabolic risk factors in men with metabolic syndrome: a randomized crossover trial. Am J Clin Nutr. (2020) 111:864-76. doi: 10.1093/ajen/nqaa026

18. Nowotny K, Jung T, Hohn A, Weber D, Grune T. Advanced glycation end products and oxidative stress in type 2 diabetes mellitus. Biomolecules. (2015) 5:194-222. doi: 10.3390/biom5010194

19. Chao CT, Huang JW, Chiang CK, Chen YC, Fang CC, Hu FC, et al. Diabetes mellitus, superoxide dismutase and peroxisome proliferator activated receptor gamma polymorphisms modify the outcome of end-stage renal disease patients of Han Chinese origin. Nephrology. (2018) 23:11725. doi: 10.1111/nep.12975

20. Kreft M. Buckwheat phenolic metabolites in health and disease. Nutr Res Rev. (2016) 29:30-9. doi: 10.1017/S0954422415000190

21. He LX, Zhao J, Huang YS Li Y. The difference between oats and beta-glucan extract intake in the management of $\mathrm{HbAlc}$, fasting glucose and insulin 
sensitivity: a meta-analysis of randomized controlled trials. Food Funct. (2016) 7:1413-28. doi: 10.1039/C5FO01364J

22. Yu K, Ke MY Li WH, Zhang SQ, Fang XC. The impact of soluble dietary fibre on gastric emptying, postprandial blood glucose and insulin in patients with type 2 diabetes. Asia Pac J Clin Nutr. (2014) 23:2108. doi: 10.6133/apjen.2014.23.2.01

23. Ninomiya K, Ina S, Hamada A, Yamaguchi Y, Akao M, Shinmachi F, et al. Suppressive effect of the alpha-amylase inhibitor albumin from buckwheat (fagopyrum esculentum moench) on postprandial hyperglycaemia. Nutrients. (2018) 10. doi: 10.3390/nu10101503

24. Lu CL, Zheng Q, Shen Q, Song C, Zhang ZM. Uncovering the relationship and mechanisms of Tartary buckwheat (Fagopyrum tataricum) and Type II diabetes, hypertension, and hyperlipidemia using a network pharmacology approach. PeerJ. (2017) 5:e4042. doi: 10.7717/peerj.4042

25. Louis S, Tappu RM, Damms-Machado A, Huson DH, Bischoff SC. Characterization of the Gut Microbial Community of Obese Patients Following a Weight-Loss Intervention Using Whole Metagenome Shotgun Sequencing. PLoS ONE. (2016) 11:e0149564. doi: 10.1371/journal.pone.0149564

26. Khanna S, Tosh PK. A clinician's primer on the role of the microbiome in human health and disease. Mayo Clin Proc. (2014) 89:107-14. doi: 10.1016/j.mayocp.2013.10.011

27. Li WZ, Stirling K, Yang JJ, Zhang L. Gut microbiota and diabetes: from correlation to causality and mechanism. World J Diabetes. (2020) 11:293308. doi: 10.4239/wjd.v11.i7.293

28. Qin J, Li Y, Cai Z, Li S, Zhu J, Zhang F, et al. A metagenome-wide association study of gut microbiota in type 2 diabetes. Nature. (2012) 490:5560. doi: 10.1038/nature11450

29. Ejtahed HS, Soroush AR, Angoorani P, Larijani B, Hasani-Ranjbar S. Gut microbiota as a target in the pathogenesis of metabolic disorders: a new approach to novel therapeutic agents. Horm Metab Res. (2016) 48:34958. doi: 10.1055/s-0042-107792

30. Yuan Y, Zhou J, Zheng Y, Xu Z, Li Y, Zhou S, et al. Beneficial effects of polysaccharide-rich extracts from Apocynum venetum leaves on hypoglycemic and gut microbiota in type 2 diabetic mice. Biomed Pharmacother. (2020) 127:110182. doi: 10.1016/j.biopha.2020.110182

31. Adeshirlarijaney A, Gewirtz AT. Considering gut microbiota in treatment of type 2 diabetes mellitus. Gut Microbes. (2020) 11:253-64. doi: 10.1080/19490976.2020.1717719

32. Lappi J, Kolehmainen M, Mykkanen H, Poutanen K. Do large intestinal events explain the protective effects of whole grain foods against type 2 diabetes? Crit Rev Food Sci Nutr. (2013) 53:631-40. doi: 10.1080/10408398.2010.5 50388

33. Xue B, Xie J, Huang J, Chen L, Gao L, Ou S, et al. Plant polyphenols alter a pathway of energy metabolism by inhibiting fecal Bacteroidetes and Firmicutes in vitro. Food Funct. (2016) 7:1501-7. doi: 10.1039/C5FO01438G

34. Calinoiu LF, Vodnar DC. Whole grains and phenolic acids: a review on bioactivity, functionality, health benefits and bioavailability. Nutrients. (2018) 10. doi: 10.3390/nu10111615
35. Gomes AC, Bueno AA, de Souza RG, Mota JF. Gut microbiota, probiotics and diabetes. Nutr J. (2014) 13:60. doi: 10.1186/1475-2891-13-60

36. Amar J, Burcelin R, Ruidavets JB, Cani PD, Fauvel J, Alessi MC, et al. Energy intake is associated with endotoxemia in apparently healthy men. Am J Clin Nutr. (2008) 87:1219-23. doi: 10.1093/ajcn/87.5.1219

37. Gurung M, Li Z, You H, Rodrigues R, Jump DB, Morgun A, et al. Role of gut microbiota in type 2 diabetes pathophysiology. EBioMedicine. (2020) 51:102590. doi: 10.1016/j.ebiom.2019.11.051

38. Yoo JY, Groer M, Dutra SVO, Sarkar A, McSkimming DI. Gut microbiota and immune system interactions. Microorganisms. (2020) 8. doi: 10.3390/microorganisms8101587

39. Zhao L, Zhang F, Ding X, Wu G, Lam YY, Wang X, et al. Gut bacteria selectively promoted by dietary fibers alleviate type 2 diabetes. Science. (2018) 359:1151-6. doi: 10.1126/science.aao5774

40. Vinolo MA, Rodrigues HG, Nachbar RT, Curi R. Regulation of inflammation by short chain fatty acids. Nutrients. (2011) 3:858-76. doi: 10.3390/nu3100858

41. Vergidis P, Ariza-Heredia EJ, Nellore A, Kotton CN, Kaul DR, Morris MI, et al. Rhodococcus infection in solid organ and hematopoietic stem cell transplant recipients ${ }^{1}$. Emerg Infect Dis. (2017) 23:510-2. doi: 10.3201/eid2303.160633

42. Yoshida N, Emoto T, Yamashita T, Watanabe H, Hayashi T, Tabata T, et al. Bacteroides vulgatus and bacteroides dorei reduce gut microbial lipopolysaccharide production and inhibit atherosclerosis. Circulation. (2018) 138:2486-98. doi: 10.1161/CIRCULATIONAHA.118.033714

43. Kim SH, Huh CS, Choi ID, Jeong JW, Ku HK, Ra JH, et al. The anti-diabetic activity of Bifidobacterium lactis HY8101 in vitro and in vivo. J Appl Microbiol. (2014) 117:834-45. doi: 10.1111/jam.12573

44. Boullier S, Tanguy M, Kadaoui KA, Caubet C, Sansonetti P, Corthesy B, et al. Secretory IgA-mediated neutralization of Shigella flexneri prevents intestinal tissue destruction by down-regulating inflammatory circuits. J Immunol. (2009) 183:5879-85. doi: 10.4049/jimmunol.0901838

Conflict of Interest: The authors declare that the research was conducted in the absence of any commercial or financial relationships that could be construed as a potential conflict of interest.

Publisher's Note: All claims expressed in this article are solely those of the authors and do not necessarily represent those of their affiliated organizations, or those of the publisher, the editors and the reviewers. Any product that may be evaluated in this article, or claim that may be made by its manufacturer, is not guaranteed or endorsed by the publisher.

Copyright (c) $2021 \mathrm{Liu}$, Yin, Hou, Liu, Ma, Zhang, Xu, Xie, Li and Wang. This is an open-access article distributed under the terms of the Creative Commons Attribution License (CC BY). The use, distribution or reproduction in other forums is permitted, provided the original author(s) and the copyright owner(s) are credited and that the original publication in this journal is cited, in accordance with accepted academic practice. No use, distribution or reproduction is permitted which does not comply with these terms. 